Pesq. Vet. Bras. 38(4):635-641, abril 2018

\title{
Encefalopatia hepática secundária à intoxicação por Tephrosia cinerea em ovinos ${ }^{1}$
}

\author{
Aline M. Silveira ${ }^{2 *}$, Édipo C. Moreira² ${ }^{2}$ Fernando $\mathrm{Vaz}^{2}$, Cíntia de Lorenzo ${ }^{3}$, \\ David Driemeier ${ }^{3}$, Fernando Furlan ${ }^{4}$, Antonio F.M. Dantas ${ }^{2}$ e Franklin Riet-Correa ${ }^{2,5}$
}

\begin{abstract}
Silveira A.M., Moreira E.C., Vaz F., Lorenzo C., Driemeier D., Furlan F., Dantas A.F.M. \& Riet-Correa F. 2018. [Hepatic encephalopathy secondary to poisoning by Tephrosia cinerea in sheep.] Encefalopatia hepática secundária à intoxicação por Tephrosia cinerea em ovinos. Pesquisa Veterinária Brasileira 38(4):635-641. Hospital Veterinário, Universidade Federal de Campina Grande, Avenida Universitária s/n, Santa Cecília, Patos, PB 58700-970, Brazil. E-mail: franklinrietcorrea@gmail.com

In the semiarid region of northeastern Brazil, Tephrosia cinerea causes periacinar hepatic fibrosis in sheep with severe ascites and, occasionally, nervous signs. Sixteen sheep from six outbreaks of T. cinerea poisoning were studied. All sheep had histologic lesion of periacinar fibrosis and six showed, in the brain, vacuolization (spongy degeneration) of the white matter and junction between grey and white matter and presence of Alzheimer type II astrocytes in the grey matter. The disease was produced experimentally in two sheep, that presented porto-sistemic shunts and similar histologic lesions as those observed in the spontaneous cases. Immunohistochemistry revealed weak labelling with anti-GFAP antibodies suggesting a degenerative alteration of astrocytes with accumulation of dense bodies and reduction of the GFAP. There was strong labelling with anti-S100 antibodies suggesting cellular reactivity with proliferation of mitochondria and endoplasmatic reticulum. Such alterations are characteristic of the effects caused by ammonia on the astrocytes. It is concluded that in poisoning by T. cinerea nervous signs due to hepatic encephalopathy occur in some sheep.
\end{abstract}

INDEX TERMS: Poisonous plants, hepatic encephalopathy, plant poisoning, Tephrosia cinerea, sheep, hepatotoxic plants, neurological disorders, status spongiosus, GFAP, S100, toxicoses.

RESUMO.- A intoxicação por Tephrosia cinerea causa fibrose hepática periacinar em ovinos na região semiárida do Nordeste, com quadro clínico de ascite acentuada, e, ocasionalmente, com sinais neurológicos. Neste trabalho foram estudadas 16 ovinos em 6 surtos de intoxicação por T. cinerea. Todos os ovinos apresentaram lesões histológicas de fibrose periacinar e seis apresentaram, no encéfalo, vacuolização da substância branca e da junção entre a substância branca e a cinzenta com

\footnotetext{
${ }^{1}$ Recebido em 28 de abril de 2017.

Aceito para publicação em 17 de maio de 2017.

${ }^{2}$ Hospital Veterinário, Universidade Federal de Campina Grande (UFCG), Avenida Universitária s/n, Santa Cecília, Patos, PB 58700-970, Brasil.

${ }^{3}$ Setor de Patologia Veterinária, Hospital Veterinário, Universidade Federal do Rio Grande do Sul (UFRGS), Av. Bento Gonçalves 9090, Agronomia, Porto Alegre, RS 90540-000, Brasil.

${ }^{4}$ Universidade Federal de Mato Grosso (UFMT), Av. Fernando Corrêa da Costa 2367, Boa Esperança, Cuiabá, MT 78060-900, Brasil.

${ }^{5}$ Instituto Nacional de Investigación Agropecuaria (INIA), La Estanzuela, Colonia, Uruguay. *Autor para correspondência: frcorrea@inia.org.uy, franklinrietcorrea@gmail.com
}

presença de astrócitos de Alzheimer tipo II na substância cinzenta. A doença foi reproduzida experimentalmente em dois ovinos que apresentaram ascite, desvios vasculares (shunts) porto-sistêmicos e sinais nervosos com lesões histológicas semelhantes a dos casos espontâneos. Na técnica de imuno-histoquímica houve marcação fraca ou ausente do citoplasma astrocitário para o anticorpo anti-GFAP em seis ovinos evidenciando uma alteração degenerativa, em que os astrócitos acumulam corpos densos e reduzem o volume de GFAP. Houve marcação positiva para o anticorpo anti-S100 em oito ovinos, incluindo os dois ovinos experimentais o que sugere reatividade celular, com proliferação mitocondrial e de retículo endoplasmático liso. Estas alterações são caraterísticas dos efeitos da amônia nos astrócitos. Conclui-se que na intoxicação por T. cinerea em alguns ovinos ocorrem sinais nervosos em consequência da encefalopatia hepática.

TERMOS DE INDEXAÇÃO: Plantas tóxicas, encefalopatia hepática, Tephrosia cinerea, ovinos, plantas hepatotóxicas, distúrbios neurológicos, degeneração esponjosa, GFAP, S100, intoxicação por plantas, toxicoses. 


\section{INTRODUÇÃo}

Tephrosia cinerea é uma planta invasora frequente na região nordeste do Brasil. As épocas de seca causam intoxicação em ovinos que pastoreiam áreas invadidas por esta planta (Santos et al. 2007). A doença se caracteriza por fibrose hepática periacinar, com hipertensão portal e aumento da pressão hidrostática intra-hepática, provocando ascite, o que faz com que os produtores conheçam a doença como "barriga d'água" (Riet-Correa et al. 2013).

A encefalopatia hepática (EH) é uma alteração clínica decorrente de insuficiência hepática. Lesões hepáticas graves causam decréscimo na metabolização da amônia, dentre outras substâncias, que pela via hematógena atinge o encéfalo causando vacuolização na substância branca (status spongiosus) e presença de astrócitos Alzheimer tipo II em diversas regiões encefálicas (Wouters et al. 2013, Kinde et al. 2014). As doenças hepáticas graves em animais de produção, principalmente algumas intoxicações por plantas, são as principais causas da encefalopatia hepática, incluindo a intoxicação por Senecio spp. em ovinos (Ilha et al. 2001) e bovinos (Rissi et al. 2010), a intoxicação por Trema micrantha em equinos (Bandarra et al. 2011) e caprinos (Traverso et al. 2003) e a intoxicação por Crotalaria retusa em equinos (Nobre et al. 2004). Não há relatos na literatura acerca da EH secundária à intoxicação por Tephrosia cinerea em ovinos; no entanto, alguns ovinos apresentam sinais nervosos e em estudos histológicos de alguns casos espontâneos se observaram lesões características desta alteração.

O objetivo deste trabalho foi estudar os casos de encefalopatia hepática secundária à intoxicação espontânea por T. cinerea, caracterizando seus aspectos histoquímicos e imuno-histoquímicos, além da reprodução experimental dessas alterações encefálicas.

\section{MATERIAL E MÉTODOS}

Foram estudados seis surtos de intoxicação natural por Tephrosia cinerea em ovinos diagnosticados no Laboratório de Patologia Animal (LPA), Hospital Veterinário, Universidade Federal de Campina Grande (UFCG), Campus de Patos, Paraíba, entre os anos de 2003 e 2016. Dados epidemiológicos, sinais clínicos e período de ocorrência dos surtos foram analisados a partir da revisão dos prontuários clínicos, identificando os casos de ovinos com lesão encefálica. Para melhor descrição e identificação da distribuição da lesão encefálica, novos cortes histológicos foram realizados.

Para a reprodução experimental da doença, T. cinerea foi colhida de julho a setembro de 2015 em uma propriedade do município de Patos, Paraíba, onde tinha ocorrido um surto de intoxicação por essa planta no ano de 2012. Após a coleta, a planta foi secada à sombra por um período de sete dias, triturada e armazenada em sacos plásticos à temperatura ambiente. Foram selecionados três ovinos, mestiços, oriundos de propriedades livres de T. cinerea. Os Ovinos E1 (10 meses, $23,1 \mathrm{~kg}$ ) e E2 ( 9 meses, $21 \mathrm{~kg}$ ) consumiram a planta ad libitum, por um período de 30 e 96 dias respectivamente. O Ovino E3 (9 meses, 23kg) foi utilizado como controle e recebeu feno de capim tifton (Cynodon dactylon) ad libitum. Os três ovinos receberam, adicionado ao volumoso, concentrado em quantidade equivalente a $1 \%$ do peso vivo e água à vontade.

Durante o período experimental, os ovinos foram alojados em baias individuais e eram submetidos à avaliação clínica diariamente. Amostras de sangue foram coletadas no primeiro dia do experimento e a cada sete dias durante todo o período experimental. As avaliações das atividades séricas de $\gamma$-glutamil transferase (GGT), aspartato aminotransferase (AST), e dos níveis séricos de albumina e ureia foram realizadas pelo método cinético enzimático e colorimétrico, de acordo com metodologia descrita no analisador bioquímico automático Roche ${ }^{\circledR}$, modelo Cobas c-111.

Os Ovinos E1 e E2 foram necropsiados após a morte natural (E1) ou após eutanásia in extremis (E2). 0 Ovino E3, controle, foi submetido à eutanásia no 100 o dia após o início do experimento. Fragmentos de fígado, pré-estômagos, abomaso, intestinos, rim, adrenal, linfonodos, pulmão e coração, assim como o sistema nervoso central foram fixados em formol tamponado a 10\% para confecção de lâminas histológicas e corados por Hematoxilina e Eosina (HE). Após a fixação foram realizados cortes transversais do encéfalo e amostras das regiões de córtex, núcleos da base, hipocampo, tálamo, colículos, pedúnculos cerebelares, cerebelo e ponte foram processadas rotineiramente para histologia e coradas por HE. Lâminas dos fígados dos ovinos intoxicados espontaneamente e experimentalmente foram coradas pelo tricrômico de Masson para evidenciar tecido fibroso. Em lâminas do encéfalo foram realizadas colorações de PAS para confirmação da integridade vascular. Foram selecionadas secções dos ovinos intoxicados naturalmente e experimentalmente e do ovino controle para o exame de imuno-histoquímica (IHQ), pela técnica estreptavidina-biotina-peroxidase (LSAB-HRP), utilizando os anticorpos anti-proteínaglial fibrilar ácida (GFAP) e anti-proteínaS100 (Quadro 1). Como controle negativo foram utilizadas as mesmas seções a serem testadas, com a substituição do anticorpo primário por diluente de anticorpo.

\section{RESULTADOS}

Os dados epidemiológicos dos seis surtos de intoxicação por Tephrosia cinerea apresentam-se no Quadro 2. Foram avaliados 16 ovinos (№ 1-16) intoxicados espontaneamente. Todos apresentaram um quadro acentuado de ascite e dispneia, com evolução clínica de 30 a 90 dias. Sinais neurológicos foram observados no final do curso clínico. Três ovinos (№ 6, 15 e 16) apresentaram embotamento seguido de ataxia, dificuldade de manter-se em estação e pressão da cabeça contra objetos; três mostravam embotamento e decúbito esternal (№ 9, 11 e 12) e apenas um apresentou vocalização (№ 11).

Durante a necropsia dos 16 ovinos intoxicados naturalmente havia, na cavidade abdominal, acentuado volume de líquido amarelado, translúcido e com filamentos de fibrina. O fígado estava recoberto por fibrina, a superfície capsular apresentava-se irregular com áreas claras deprimidas e a

Quadro 1. Origem dos anticorpos primários, diluições, recuperações antigênicas e cromógeno utilizados na imunohistoquímica

\begin{tabular}{ccccc}
\hline $\begin{array}{c}\text { Anticorpo } \\
\text { primário }^{\mathrm{a}}\end{array}$ & $\begin{array}{c}\text { Especificações } \\
\text { do anticorpo }\end{array}$ & $\begin{array}{c}\text { Diluição do } \\
\text { anticorpo }\end{array}$ & $\begin{array}{c}\text { Recuperação } \\
\text { antigênica }\end{array}$ & Cromógeno \\
\hline Anti-GFAP & Policlonal & $1: 500$ & TRIS-EDTA $^{\mathrm{c}}$ & DAB $^{\mathrm{d}}$ \\
(Ref. Z0334) & coelho & & $\begin{array}{c}\text { pH 9.0, } 96^{\circ} \mathrm{C}, \\
10 \mathrm{~min}\end{array}$ & \\
& & & Tampão & DAB \\
Anti-S100 & Policlonal & \multirow{2}{*}{$1: 200$} & citrato pH & \\
(Ref. Z0311) & coelho & & $6.0,96^{\circ} \mathrm{C}$, & \\
& & & $20 \mathrm{~min}$ & \\
& & & &
\end{tabular}

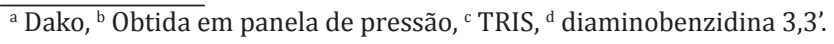


superfície de corte evidenciava padrão lobular evidente. Adicionalmente, nos Ovinos 4 e 16, havia desvios vasculares (shunts) porto-sistêmicos (Fig.1A), os quais consistiam de numerosos vasos tortuosos de pequeno calibre, originados nos ramos abdominais da veia porta que anastomosavam-se na veia cava abdominal.
Microscopicamente, observou-se, no fígado, leve a acentuada proliferação de fibroblastos, na região periacinar, formando pontes, associada, por vezes, à moderada hemorragia. Além disso, havia fibrose capsular que infiltrava o parênquima hepático (Fig.1B). Ao ser analisado o sistema nervoso dos 16 ovinos, notou-se que os № 2, 5, 11, 12, 15 e 16 (37,5\%), apresentaram

Quadro 2. Dados epidemiológicos dos ovinos intoxicados naturalmente por Tephrosia cinerea

\begin{tabular}{|c|c|c|c|c|c|c|c|c|c|c|}
\hline Surto & Local & Data & $\begin{array}{l}\text { Total de } \\
\text { animais }\end{array}$ & № Doentes & № Mortos ${ }^{\mathrm{a}}$ & № & $\begin{array}{c}\text { Tipo de } \\
\text { morte }\end{array}$ & Idade & Raça & Sexo \\
\hline \multirow[t]{4}{*}{1} & São José do Bomfim, PB & Nov a Dez 2003 & 35 & 10 & 8 & 1 & Natural & 8 meses & Santa Inês & $\mathrm{F}^{\mathrm{b}}$ \\
\hline & & & & & & 2 & Natural & 3 anos & $\mathrm{SRD}^{\mathrm{b}}$ & $\mathrm{F}$ \\
\hline & & & & & & 3 & Natural & Adulta & Santa Inês & $\mathrm{F}$ \\
\hline & & & & & & 4 & Natural & Adulta & Santa Inês & $\mathrm{F}$ \\
\hline 2 & Caicó, RN & Nov 2004 & 10 & 1 & 1 & 5 & Eutanásia & 1 ano & SRD & $\mathrm{M}^{\mathrm{b}}$ \\
\hline \multirow[t]{2}{*}{3} & Serra Negra, RN & Ago 2007 & 150 & 30 & 23 & 6 & Natural & 6 meses & SRD & $\mathrm{F}$ \\
\hline & & & & & & 7 & Eutanásia & 6 meses & SRD & $\mathrm{F}$ \\
\hline 4 & Patos, PB & Out 2008 & 60 & 30 & 1 & 8 & Natural & 6 meses & Dorper & $\mathrm{F}$ \\
\hline \multirow[t]{2}{*}{5} & Catingueira, PB & Set 2010 & 80 & $\mathrm{NI}^{\mathrm{b}}$ & $\mathrm{NI}$ & 9 & Natural & $\mathrm{NI}$ & SRD & $\mathrm{F}$ \\
\hline & & & & & & 10 & Natural & 8 meses & SRD & $\mathrm{F}$ \\
\hline \multirow[t]{3}{*}{6} & São José do Bomfim, PB & Mai a Nov & 50 & 20 & 20 & 11 & Natural & Jovem & SRD & $\mathrm{F}$ \\
\hline & & 2012 & & & & 12 & Eutanásia & Adulto & SRD & $\mathrm{F}$ \\
\hline & & & & & & 13 & Natural & Adulto & SRD & M \\
\hline 7 & Patos, PB & Set 2012 & 35 & 3 & 2 & 14 & Natural & 6 anos & Dorper & $\mathrm{F}$ \\
\hline 8 & Catingueira, PB & Out 2012 & 40 & $\mathrm{NI}$ & $\mathrm{NI}$ & 15 & Natural & 3 anos & Dorper & $\mathrm{M}$ \\
\hline 9 & Patos, PB & Set 2015 & 25 & 3 & 0 & 16 & Eutanásia & 1 ano & Santa Inês & $\mathrm{F}$ \\
\hline
\end{tabular}

a Número de mortes que ocorreram antes ou durante as visitas, não foi informado se os animais que permaneceram doentes morreram posteriormente,

${ }^{\mathrm{b}} \mathrm{NI}=$ não informado, $\mathrm{SRD}=$ sem raça definida, $\mathrm{M}=$ macho, $\mathrm{F}=$ fêmea.

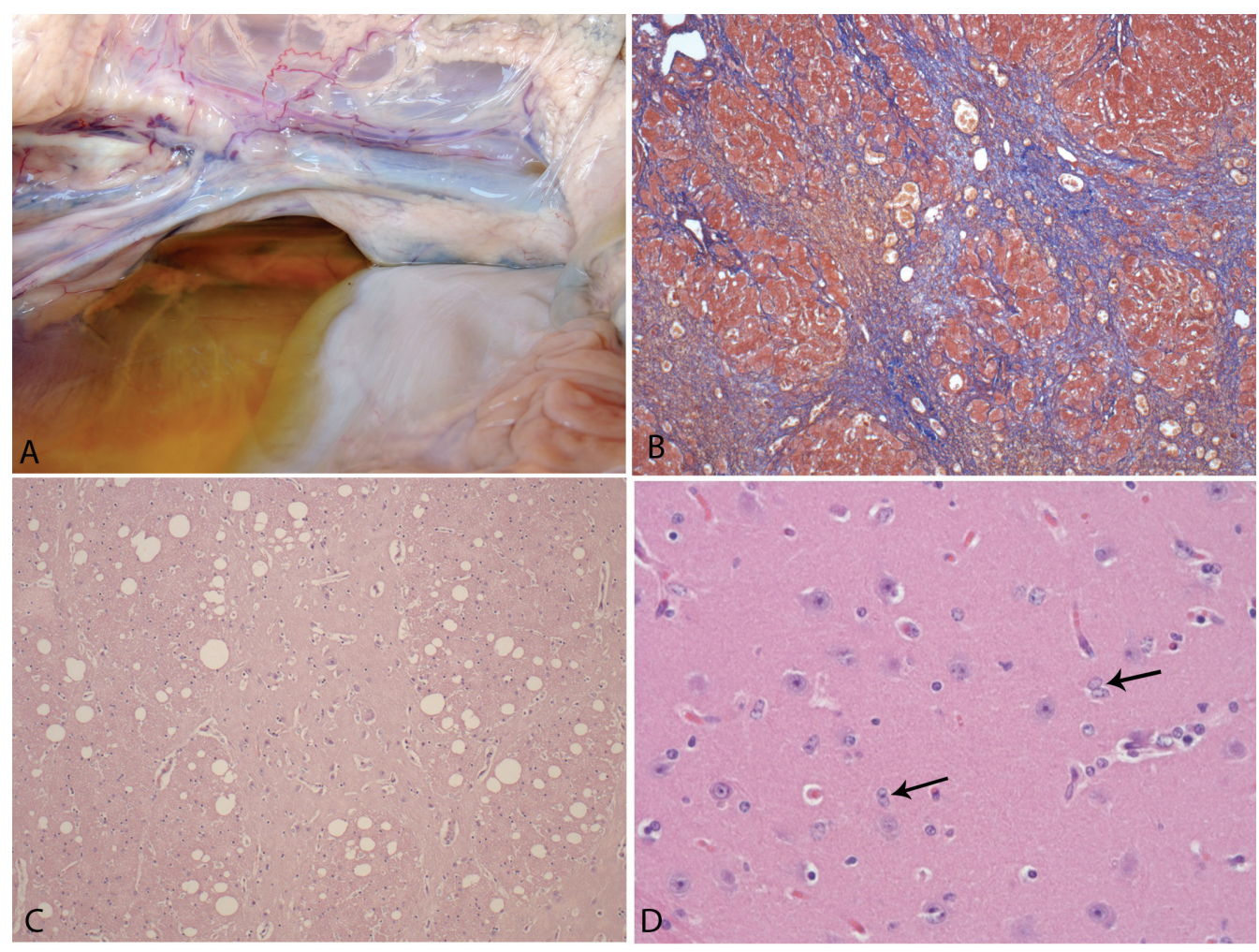

Fig.1. (A) Cavidade abdominal de Ovino 16, intoxicado por Tephrosia cinerea, com líquido ascítico livre na cavidade e presença de shunts porto-sistêmicos. (B) Fígado do Ovino 2, intoxicado por T. cinerea, com acentuada fibrose periacinar e formação de pontes. Tricômico de Masson, obj.4x. (C) Junção entre substância branca e cinzenta de telencéfalo do Ovino 12, intoxicado por T. cinerea, com marcada vacuolização nas junções entre as substâncias branca e cinzenta. HE, obj.20x. (D) Substância cinzenta de córtex telencefálico do Ovino 2, intoxicado por T. cinerea, evidenciando astrócitos de Alzheimer tipo II (seta). HE, obj.40x. 
leve a acentuada vacuolização na junção entre a substância branca e a cinzenta, e na substância branca, principalmente nas porções ventrais das regiões de pedúnculo cerebelar, colículos, cápsula externa e interna, tálamo e hipocampo. Os vacúolos possuíam formato redondo a ovalado e apareciam isolados ou unidos, formando uma sequência linear (Fig.1C). Os Ovinos 2, 12, 15 e 16 exibiram astrócitos de Alzheimer tipo II (AAII) na substância cinzenta da região telencefálica cortical, tálamo, hipocampo e colículos. Estas células eram caracterizadas por núcleo grande, angulado e cromatina pouco condensada; estavam dispostos isolados ou em grupos de dois a quatro, distribuídos de forma aleatória e próximos a corpos de neurônios (Fig.1D).

Os exames bioquímicos dos ovinos intoxicados experimentalmente (Fig.2) mostraram atividades séricas elevadas de GGT e, no dia da morte, aumento das atividades séricas de AST. A albumina não demonstrou alterações significativas. 0 Ovino E1, 28 dias após o início da ingestão da planta, apresentou apatia e aumento bilateral de volume abdominal. No teste de balotamento constatou-se líquido abdominal, que foi drenado por abdominocentese e apresentava-se inodoro, translúcido, discretamente amarelado, com escassos filamentos de fibrina. Adicionalmente, o rúmen apresentava-se com meteorismo, consistência macia à palpação e atonia. 0 ovino apresentava ainda embotamento e anorexia. No dia seguinte, apresentou diarreia, com fezes
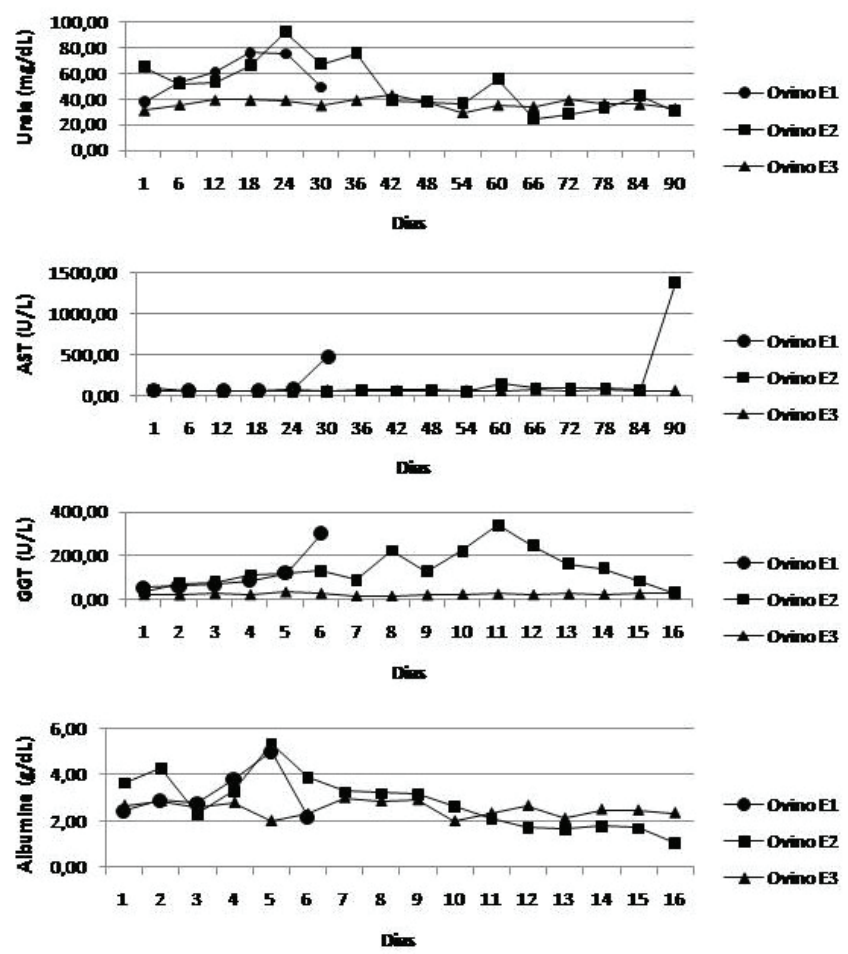

Fig.2. Níveis de albumina, ureia, GGT e AST do grupo experimental de ovinos intoxicados por Tephrosia cinerea e grupo controle. Os valores de referência normal para ureia são $12-42,8 \mathrm{mg} / \mathrm{dL}$, para AST 60-280 U/L, para GGT 20-52 U/L e para albumina $2,4-3 \mathrm{~g} / \mathrm{dL}$. amareladas com presença de sangue. Após 30 dias do início do experimento exibiu ataxia, dificuldade em manter-se em estação e morreu naturalmente no mesmo dia. A necropsia foi realizada imediatamente após a morte. Havia dois litros de líquido livre na cavidade abdominal. 0 fígado estava com a superfície capsular irregular, com áreas deprimidas, esbranquiçadas, multifocais a coalescentes, entremeadas por áreas avermelhadas, além de marcada evidenciação do padrão lobular. Ao corte este padrão se estendia ao parênquima do órgão. Havia formação de shunts porto-sistêmicos, entre os vasos mesentéricos, veia porta e veia cava abdominal, além de marcado edema no mesentério, abomaso e vesícula biliar.

O Ovino E2 apresentou hiporexia a partir do 54으 dia da ingestão da planta. No 67을 dia notou-se distensão bilateral abdominal, caracterizada como ascite no teste de balotamento. No $78^{\circ}$ dia procedeu-se a coleta e análise do líquido que evidenciou as mesmas características do líquido coletado do Ovino E1. Oitenta e cinco dias após o início do experimento, o Ovino E2 apresentava-se com um quadro clínico de embotamento e redução dos reflexos de dor e de resposta a ameaça. No 89 dia mantinha a cabeça pressionada contra a parede da baia e um dia após permaneceu em decúbito esternal, com ausência de resposta a estímulos externos. No dia seguinte, devido suas condições clínicas, foi eutanasiado e realizada a necropsia. Na cavidade abdominal, havia 20 litros de fluido com as mesmas características observadas no Ovino E1. As lesões do fígado eram semelhantes às do Ovino E1, porém, mais evidentes e numa extensão maior. A parede do abomaso e dos pré-estômagos estava edemaciada, assim como o mesentério.

Microscopicamente, no fígado dos dois ovinos havia fibrose periacinar associada à moderada hemorragia. Ao redor destas regiões alguns hepatócitos apresentavam-se necróticos, com eosinofilia citoplasmática e picnose nuclear. Ocasionalmente, nessas células, havia corpúsculos apoptóticos eosinofílicos intracitoplasmáticos. Nos cortes do sistema nervoso central foram observadas lesões semelhantes às dos ovinos intoxicados espontaneamente.

Através do estudo histoquímico, evidenciou-se nos ovinos intoxicados espontânea e experimentalmente, que as alterações hepáticas eram evidenciadas principalmente nas secções coradas pelo Tricrômio de Masson. Nas secções coradas pelo método do PAS evidenciou-se integridade da membrana vascular dos capilares encefálicos, mesmo em regiões adjacentes às áreas de vacuolização. Um resumo das principais lesões hepáticas e encefálicas dos ovinos espontaneamente intoxicados por T. cinerea está disposto no Quadro 3.

Na IHQ com o anticorpo anti-GFAP notou-se fraca ou nenhuma marcação dos astrócitos de Alzheimer tipo II, no córtex e colículo dos Ovinos 2, 6, 12 e 16 (Fig.3) e nos ovinos experimentais. Com o anticorpo anti-S100 houve imunomarcação em todos os cortes, variando de fraca nos Ovinos 2, 12 e 15, a moderada nos Ovinos 6, 11, 16 e nos ovinos experimentais. Os demais ovinos demonstraram marcação idêntica ao controle no uso dos dois anticorpos (Fig.4). Não foi constatada correlação entre o grau de fibrose hepática e o grau de lesão encefálica (Quadro 3). 
Quadro 3. Grau da fibrose no fígado e presença de astrócitos de Alzheimer tipo II e vacuolização no encéfalo de ovinos intoxicados naturalmente por Tephrosia cinerea

\begin{tabular}{|c|c|c|c|}
\hline \multirow{2}{*}{ № Ovino } & \multirow{2}{*}{ Fibrose hepática } & \multicolumn{2}{|c|}{ Lesão encefálica } \\
\hline & & AAII $^{\mathrm{a}}$ & Vacuolização \\
\hline 2 & +++ & $+++\mathrm{HC}, \mathrm{NB}, \mathrm{CT}$ & $+\mathrm{TA}$ \\
\hline 4 & +++ & - & - \\
\hline 5 & + & - & - \\
\hline 6 & + & $++\mathrm{NB}, \mathrm{PC}$ & $++\mathrm{NB}, \mathrm{TA}, \mathrm{PC}, \mathrm{CO}$ \\
\hline 9 & +++ & - & - \\
\hline 10 & + & - & - \\
\hline 11 & + & - & $+++C T, T A, N B, P C, C B, C O$ \\
\hline 12 & + & $++\mathrm{CT},+++\mathrm{NB}$ & $+++\mathrm{CT}, \mathrm{TA}, \mathrm{NB}, \mathrm{PC}$ \\
\hline 13 & ++ & - & - \\
\hline
\end{tabular}

${ }^{a}$ Astrócitos Alzheimer tipo II, - ausente, + pouca quantidade, ++ moderada quantidade, +++ grande quantidade $. \mathrm{CB}=$ cerebelo, $\mathrm{CT}=$ córtex, $\mathrm{CO}=$ colículos, $\mathrm{HC}=$ hipocampo, $\mathrm{NB}=$ : núcleos da base, $\mathrm{PC}=$ pedúnculo cerebelar, $\mathrm{TA}=$ tálamo.

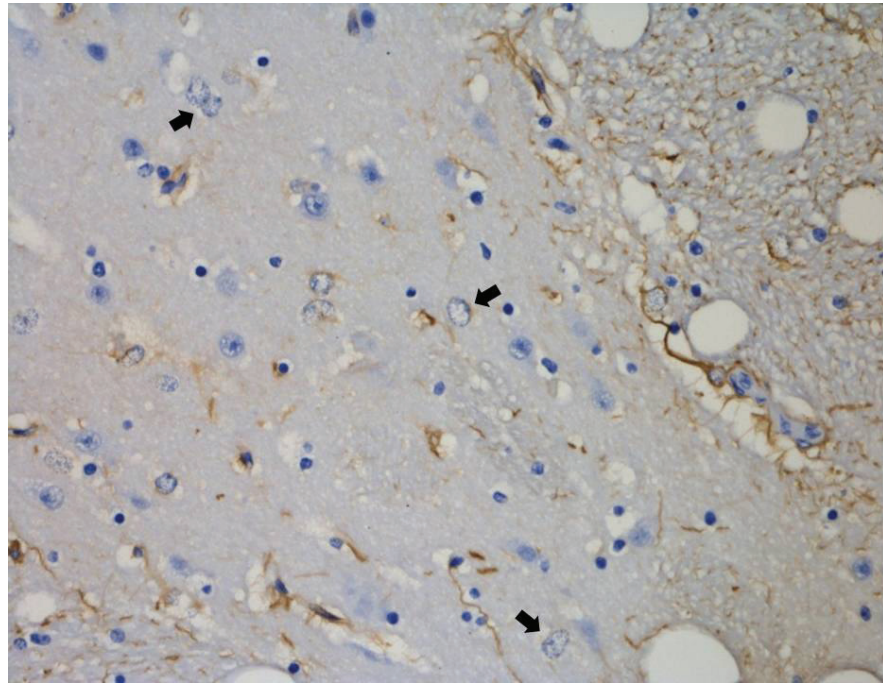

Fig.3. Junção entre substância branca e substância cinzenta de telencéfalo do Ovino 6, intoxicado por Tephrosia cinerea, com astrócitos de Alzheimer tipo II com fraca ou ausência de marcação citoplasmática (setas). Imunomarcação anti-GFAP, método estreptavidina-biotinaperoxidase. Cromógeno DAB, obj.40x.

\section{DISCUSSÃO}

Os sinais clínicos neurológicos dos ovinos intoxicados espontaneamente e experimentalmente foram observados somente no final do curso clínico da doença; portanto, é provável que alguns ovinos afetados espontaneamente, que não apresentaram sinais neurológicos, tenham sido eutanasiados antes de apresentarem lesões no sistema nervoso central. 0 mesmo pode ter ocorrido nos casos experimentais reportados anteriormente, que não apresentaram sinais neurológicos ou lesões do sistema nervoso (Santos et al. 2007, Riet-Correa et al. 2013), provavelmente, por terem sido eutanasiados antes da apresentação dos sinais nervosos.

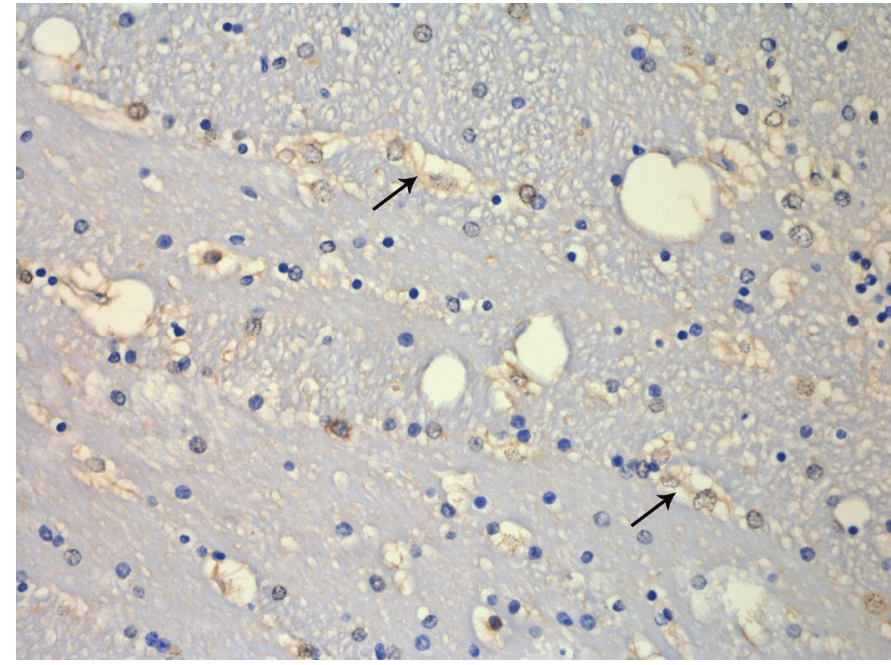

Fig.4. Junção entre substância branca e substância cinzenta de telencéfalo do Ovino 12, intoxicado por Tephrosia cinerea. Imunomarcação anti-S100 em astrócitos (seta), método estreptavidina-biotinaperoxidase. Cromógeno DAB, obj.40x.

Nos ovinos experimentais observou-se que o quadro neurológico é semelhante à ao observado na intoxicação por Senecio brasiliensis em ovinos (Ilha et al. 2001) e bovinos (Wouters et al. 2013). Entretanto, quando se compara a intensidade dessa sintomatologia clínica com casos experimentais de infusão intravenosa de amônia em ovinos, nota-se que nestes últimos os sinais clínicos são mais acentuados e diversificados, com presença de convulsão e pitialismo (Hooper \& Finnie 1987).

A elevação dos níveis de AST ao final do experimento pode ser devida a lesão dos hepatócitos ou, no caso do Ovino E2, poderia estar associada à lesão muscular, devido ao decúbito 
(Tennant \& Center 2008). 0 padrão da GGT, em ambos ovinos, reflete uma lesão acentuada no fígado afetando também a região periportal (Tennant \& Center 2008). A ausência de alterações da albumina sérica confirma que a ascite nos casos de intoxicação por Tephrosia cinerea é decorrente do aumento da pressão hidrostática, devido à fibrose periacinar e capsular do fígado e não por redução da pressão oncótica (Riet-Correa et al. 2013).

Nos ovinos estudados houve redução da concentração da ureia sérica que pode ter ocorrido pela não metabolização da amônia ou pela diminuição na ingestão de alimentos. Os hepatócitos centrolobulares são mais eficazes na conversão da amônia, portanto as lesões nesta região aumentam os níveis sérios de amônia (Häussinger 1983). Além disso, os shunts porto-sistêmicos em resposta à fibrose hepática, previamente descritos nos casos de intoxicação crônica em ovinos por T. cinerea (Riet-Correa et al. 2013), acentuam a hiperamonemia. A descrição dos shunts no laudo necroscópico dos Ovinos 4 e 16 e sua visualização nos ovinos experimentais sugere esta patogenia. É provável que nos demais ovinos intoxicados naturalmente houvesse shunts porto-sistêmicos que não tenham sidos registrados na descrição das necropsias.

Histologicamente, a lesão encefálica mais frequentemente visualizada neste trabalho foi a presença de vacúolos na substância branca e junção entre a substância branca e a cinzenta de diversas regiões do encéfalo, principalmente nos pedúnculos cerebelares, colículos, tálamo e cápsula interna e externa. Essas lesões são semelhantes às descritas em bezerros com hiperamonemia induzida experimentalmente (Cho \& Leipold 1977), em bovinos intoxicados por Senecio spp. (Wouters et al. 2013) e na encefalopatia hepática de diversas espécies de animais (Stalker \& Hayes 2007).

No encéfalo, a hiperamonemia tem inicialmente um efeito citotóxico nos astrócitos que, em resposta, liberam grande quantidade de glutamina, que provoca estresse oxidativo, anormalidades na ação das mitocôndrias e distúrbios na osmolaridade celular. Essas alterações se caracterizam, histologicamente, por astrócitos grandes, vacuolizados e por vezes em grupos de dois a mais de quatro, denominados de astrócitos de Alzheimer tipo II, visualizados predominantemente em equinos com EH (Summers et al. 1994, Nobre et al. 2004, Tennant \& Center 2008). Sugere-se que a alteração encefálica ocorra inicialmente nos astrócitos e, posteriormente, um distúrbio osmótico dos oligondendrócitos, permite o influxo de líquido entre as lamelas de mielina, que é visualizado histologicamente como vacúolos na substância branca e junção entre a substância branca e cinzenta em regiões específicas do encéfalo (Summers et al. 1994, Ilha et al. 2001). A ausência de lesões vasculares, constatada na coloração de PAS, confirma que não há alterações vasculares na patogenia da EH na intoxicação crônica por T. cinerea, semelhante ao descrito na EH em bovinos na intoxicação por Senecio sp. (Wouters et al. 2013).

A amônia é capaz de provocar duas respostas astrocitárias, a primeira, trata-se de uma reatividade celular, com proliferação mitocondrial e do retículo endoplasmático liso, a qual pode ser visualizada mediante marcação positiva com o anticorpo anti-S-100 (Donato et al. 2013), fato ocorrido nos Ovinos 2, 6, 11, 12, 15, 16, e nos ovinos experimentais. A segunda resposta é uma alteração degenerativa, em que os astrócitos acumulam corpos densos e reduzem o volume de GFAP, fazendo com que haja pouca imunoreatividade ao anticorpo anti-GFAP no citoplasma dos astrócitos, como ocorreu nos Ovinos 2, 6, 12, 16 e nos ovinos experimentais. Tais alterações ocorrem pelo o efeito da amônia nos astrócitos (Summers et al. 1994) e são semelhantes às encontradas no encéfalo de bovinos (Wouters et al. 2013) e equinos intoxicados por T. micranta (Bandarra et al. 2011).

\section{CONCLUSÕES}

Tephrosia cinerea pode provocar encefalopatia hepática em ovinos, com vacuolização de substância branca e presença de astrócitos de Alzheimer tipo II, predominantemente nas regiões de núcleos da base, colículos, tálamo e córtex.

A análise do encéfalo pelo método histoquímico de PAS não demonstra lesões vasculares na patogenia da $\mathrm{EH}$.

A fraca marcação no citoplasma dos astrócitos com emprego do anticorpo anti-GFAP reflete degeneração celular e a forte marcação pelo anticorpo anti-S100 sugere reatividade destas células, caracterizando que a patogenia da EH em ovinos intoxicados por T. cinerea ocorre em decorrência de hiperamonemia com disfunção astrocitária.

Agradecimentos.- À Coordenação de Aperfeiçoamento de Pessoal de Nível Superior (CAPES) pela bolsa do primeiro autor. Trabalho financiado pelo Instituto Nacional de Ciência e Tecnologia para o Controle das Intoxicações por Plantas, Processo CNPq 573534/2008-0.

\section{REFERÊNCIAS}

Bandarra P.M., Bezerra Júnior P.S., Oliveira L.G.S., Correa F.L.F., Borba M.R., Reck Júnior J., Cruz C.E.F. \& Driemeier D. 2011. Intoxicação experimental por Trema micranta (Cannabaceae) em equinos. Pesq. Vet. Bras. 31(11):991996. http://dx.doi.org/10.1590/S0100-736X2011001100009.

Cho D.Y. \& Leipold H.W. 1977. Experimental spongy degeneration in calves. Acta Neuropathol. 39(2):115-127.http://dx.doi.org/10.1007/BF00703317. PMid:899739.

Donato R., R. Cannon B., Sorci G., Riuzzi F., Hsu K., J. Weber D. \& L. Geczy C. 2013. Functions of S100 proteins. Curr. Mol. Med. 13(1):24-57. http:// dx.doi.org/10.2174/156652413804486214. PMid:22834835.

Häussinger D. 1983. Hepatocyte heterogeneity in glutamine and ammonia metabolism and the role of an intercellular glutamine cycle during ureogenesis in perfused rat liver. Eur. J. Biochem. 133(2):269-275. http:// dx.doi.org/10.1111/j.1432-1033.1983.tb07458.x. PMid:6852039.

Hooper P.T. \& Finnie J.W. 1987. Focal spongy changes in the central nervous system of sheep and cattle. J. Comp. Pathol. 97(4):433-440. http://dx.doi. org/10.1016/0021-9975(87)90021-1. PMid:3667997.

Ilha M.R.S., Loretti A.P., Barros S.S. \& Barros C.S.L. 2001. Intoxicação espontânea por Senecio brasiliensis (Asteraceae) em ovinos no Rio Grande do Sul. Pesq. Vet. Bras. 21(3):123-138. http://dx.doi.org/10.1590/S0100736X2001000300005.

Kinde H., Pesavento P.A., Loretti A.P., Adaska J.M., Barr B.C., Moore J.D., Anderson M.L., Rimoldi G., Hill A.E. \& Jones M.E.B. 2014. Congenital portosystemic shunts and hepatic encephalopathy in goat kids in California: 11 cases (1999-2012). J. Vet. Diagn. Invest. 26(1):173-177. http://dx.doi. org/10.1177/1040638713518050. PMid:24532697.

Nobre V.M.T., Riet-Correa F., Barbosa Filho J.M., Dantas A.F.M., Tabosa I.M. \& Vasconcelos J.S. 2004. Intoxicação por Crotalariaretusa (Fabaceae) em eqüídeos no semi-árido da Paraíba. Pesq. Vet. Bras. 24(3):132-143. http:// dx.doi.org/10.1590/S0100-736X2004000300004.

Riet-Correa F., Carvalho K.S., Riet-Correa G., Barros S.S., Simões S.V.D., Soares M.P. \& Medeiros R.M.T. 2013. Periacinar liver fibrosis caused by Tephrosia 
cinerea in sheep. Res. Vet. Sci. 95(1):200-203. http://dx.doi.org/10.1016/j. rvsc.2013.03.008. PMid:23587159.

Rissi D.R., Pierezan F., Oliveira-Filho J.C., Lucena R.B., Carmo P.M.S. \& Barros C.S.L. 2010. Abordagem diagnóstica das principais doenças do sistema nervoso de ruminantes e equinos no Brasil. Pesq. Vet. Bras. 30 (11):358367. http://dx.doi.org/10.1590/S0100-736X2010001100010.

Santos J.C.A., Riet-Correa F., Dantas A.F.M., Barros S.S., Molyneux R.J., Medeiros R.M.T., Silva D.M. \& Oliveira O.F. 2007. Toxic hepatopathy in sheep associated with the ingestion of the legume Tephrosia cinerea. J. Vet. Diagn. Invest. 19(6):690-694. http://dx.doi.org/10.1177/104063870701900612. PMid:17998559.

Stalker M.J. \& Hayes M.A. 2007. Liver and biliary system, p.330-331. In: Jubb K.V.F., Kennedy P.C. \& Palmer N. (Ed) Pathology of domestic animals. Vol.2. 5th ed. Elsevier, Philadelphia.
Summers B.A., Cummings J.F. \& De Lahunta A. 1994. Degenerative diseases of the central nervous system, p.208-2011. In: Ibid. (Eds), Veterinary Neuropahology. Mosby, St Louis.

Tennant B.C. \& Center S.A. 2008. Hepatic function, p.379-412. In: Kaneko J.J., Harvey J.W. \& Bruss M.L. (Eds), Clinical Biochemistry of Domestic Animals. Elsevier, San Diego. http://dx.doi.org/10.1016/B978-0-12370491-7.00013-1.

Traverso S.D., Colodel E.M., Loretti A.P., Correia A.M. \& Driemeier D. 2003. Intoxicação natural por Trema micrantha em caprinos. Ciência Rural 33(1):133-136. http://dx.doi.org/10.1590/S0103-84782003000100021.

Wouters A.T.B., Boabaid F.M., Fredo G., Wouters F. \& Driemeier D. 2013. Caracterização imuno-hostoquímica das alterações encefálicas em bovinos com hepatopatia tóxica por Senecio sp. Pesq. Vet. Bras. 33(11):1325-1331. http://dx.doi.org/10.1590/S0100-736X2013001100007. 